Bernades, DM, Celeste, WC \& Chaves, GLD. (2020). Energy efficiency in urban public lighting: literature review of equipment and technologies. Research, Society and Development, 9(7): 1-21, e606973957.

\title{
Eficiência energética na iluminação pública urbana: revisão bibliográfica dos
} equipamentos e tecnologias

Energy efficiency in urban public lighting: literature review of equipment and technologies

Eficiencia energética en alumbrado público urbano: revisión bibliográfica de equipos y tecnologías

Recebido: 25/04/2020 | Revisado: 08/05/2020 | Aceito: 20/05/2020 | Publicado: 30/05/2020

\section{Drielly Mazzarim Bernades}

ORCID: https://orcid.org/0000-0002-3369-5721

Universidade Federal do Espírito Santo, Brasil

E-mail: driellymazzarim@gmail.com

Wanderley Cardoso Celeste

ORCID: https://orcid.org/0000-0002-1121-937X

Universidade Federal do Espírito Santo, Brasil

E-mail: wanderley.celeste@ufes.br

Gisele de Lorena Diniz Chaves

ORCID: https://orcid.org/0000-0001-6359-9063

Universidade Federal do Espírito Santo, Brasil

E-mail: gisele.chaves@ufes.br

\section{Resumo}

Com o aumento da população e a necessidade por maiores quantidades de recursos naturais para suprir toda demanda energética mundial, é fundamental a busca pelo uso sustentável desses recursos. Uma das maneiras de se obter isso é por meio da eficiência energética. Assim, o presente trabalho apresenta e compara os principais recursos existentes para melhoria da eficiência energética na iluminação pública urbana, através de pesquisa bibliográfica e documental. Paralelamente, são citados os programas de eficiência energética no Brasil e os incentivos promovidos pelas instituições ligadas ao governo. Por fim, são demonstradas as tecnologias atuais com estudos de caso que visam promover a eficiência energética da iluminação pública urbana, destacando suas vantagens e desvantagens. Como 
resultado, identifica-se que a alternativa mais viável para revitalização do sistema de iluminação pública é o retrofit das lâmpadas tradicionais por lâmpadas LED, por se tratar de um recurso com maior durabilidade, menor consumo de energia elétrica e menor necessidade de adaptações da rede elétrica atual. No entanto, a partir do retrofit com as lâmpadas de LED, é possível implantar recursos e tecnologias adicionais que permitam o monitoramento inteligente de todo sistema, acrescentando, além de maior economia de energia, mais segurança para a população.

Palavras-chave: Gestão pública; Eficiência energética; Iluminação pública; Sustentabilidade.

\begin{abstract}
With the increase in population and the need for greater amounts of natural resources to meet all the world's energy demand, the search for sustainable use of these resources is essential. One way to achieve this is through energy efficiency. Thus, this paper presents and compares the main existing resources for improving energy efficiency in urban public lighting, through bibliographic and documentary research. At the same time, energy efficiency programs in Brazil and incentives promoted by government-related institutions are cited. Finally, current technologies are demonstrated with case studies that aim to promote the energy efficiency of urban street lighting, highlighting their advantages and disadvantages. As a result, it is identified that the most viable alternative for revitalizing the street lighting system is the retrofit of traditional lamps by LED lamps, as it is a feature with greater durability, lower power consumption and less need for network adaptations. electrical current. However, from retrofit with LED lamps, it is possible to deploy additional features and technologies that allow intelligent monitoring of the entire system, adding, in addition to greater energy savings, more safety for the population.
\end{abstract}

Keywords: Public management; Energy efficiency; Public street lighting; Sustainability.

\title{
Resumen
}

Con el aumento de la población y la necesidad de mayores cantidades de recursos naturales para suplir toda la demanda mundial de energía, la búsqueda del uso sostenible de estos recursos es esencial. Una de las formas de lograr esto es a través de la eficiencia energética. Así, el presente trabajo presenta y compara los principales recursos existentes para mejorar la eficiencia energética en el alumbrado público urbano, a través de la investigación bibliográfica y documental. Al mismo tiempo, se mencionan los programas de eficiencia energética en Brasil y los incentivos promovidos por instituciones vinculadas al gobierno. 
Finalmente, las tecnologías actuales se demuestran con estudios de casos que tienen como objetivo promover la eficiencia energética del alumbrado público urbano, destacando sus ventajas y desventajas. Como resultado, se identifica que la alternativa más viable para revitalizar el sistema de alumbrado público es la actualización de las lámparas tradicionales por lámparas LED, ya que es un recurso con mayor durabilidad, menor consumo de electricidad y menos necesidad de adaptaciones de red. corriente electrica. Sin embargo, desde la actualización con lámparas LED, es posible implementar recursos y tecnologías adicionales que permiten un monitoreo inteligente de todo el sistema, agregando, además de un mayor ahorro de energía, más seguridad para la población.

Palabras clave: Gestión pública; Eficiencia energética; Alumbrado público; Sostenibilidad.

\section{Introdução}

Atualmente, uma das grandes dificuldades da humanidade é gerir o setor energético, de forma a preservar os recursos naturais, reduzir os impactos ao meio ambiente e racionalizar o consumo mundial de energia, sem prejudicar o crescimento da população e da economia. Uma das pontas desse setor é a energia elétrica que ainda é muito dependente de combustíveis fósseis, que são fontes finitas e contribuem consideravelmente para o aumento da emissão de gases de efeito estufa. Dados da IEA (International Energy Agency) de 2017 mostram que os combustíveis fósseis são responsáveis por cerca de $64 \%$ da produção mundial de eletricidade e que o consumo total mundial de eletricidade atingiu $21.372 \mathrm{TWh}$, ultrapassando em 2,6\% o consumo mundial em 2016, enquanto que a geração mundial de eletricidade foi $2,5 \%$ superior a 2016 (IEA, 2020).

Com o objetivo de mudar esse cenário, a maior parte das pesquisas na área de energia, são direcionadas à geração de energia elétrica por fontes renováveis e mais limpas, como a biomassa, a energia solar e a energia eólica (Manzano-Agugliaro et al., 2013). Além de iniciativas para promover a cultura de eficiência energética e redução de consumo energético, propostas por várias nações e organizações (Campisi, Gitto \& Morea, 2018). Com o aumento do consumo de energia, a eficiência energética se torna fundamental em todos os setores (Dapper, Tomé \& Zanatta, 2019).

A eficiência energética é um importante vetor no atendimento à demanda futura de energia, já que contribui para a segurança energética, competitividade econômica e redução de impactos ambientais. O aproveitamento de oportunidades para tal requer uma visão integrada tanto de fontes energéticas quanto dos agentes envolvidos, o que engloba a 
sociedade em geral (Kruger \& Ramos, 2016).

A eficiência energética tem se tornado uma ferramenta cada vez mais importante na sociedade atual e também está sendo aplicada na minimização do consumo de eletricidade para a iluminação pública nos municípios do Brasil por meio do PROCEL (Programa Nacional de Conservação de Energia Elétrica), que possui um subprograma chamado RELUZ, que incentiva e investe em projetos de eficiência energética na iluminação pública, substituindo equipamentos com tecnologias menos eficientes por outras de maior eficiência.

Promover a eficiência energética é utilizar o conhecimento no ramo energético de forma aplicada, empregando os conceitos da engenharia, da economia e da administração aos sistemas energéticos. Devido à diversidade e complexidade desses sistemas, é interessante apresentar técnicas e métodos para definir objetivos e ações para melhorar o desempenho energético e reduzir as perdas nos processos de transporte, armazenamento e distribuição de energia (Elektro et al., 2012).

Este trabalho tem o objetivo de apresentar e comparar alguns dos recursos disponíveis capazes de promover a eficiência energética na iluminação pública como, por exemplo, equipamentos e tecnologias baseadas em softwares e métodos, relacionando suas vantagens e desvantagens. Ainda, são relatados estudos de caso com a aplicação desses recursos na iluminação de espaços públicos, com o intuito de nortear os projetos de iluminação, focando a otimização da energia elétrica destinada a esse fim. A metodologia baseia-se em pesquisa bibliográfica e documental em fontes de publicações oficiais de órgãos do setor elétrico brasileiro e internacional, bases de dados científicos, periódicos apresentados em congressos e demais pesquisas desenvolvidas na área.

Este artigo segue com a seguinte estruturação: a Seção 2 trata da eficiência energética no Brasil; a Seção 3 discorre sobre os principais recursos de iluminação pública, bem como as tecnologias aplicadas nos projetos de iluminação pública; e, por fim, a Seção 4 apresenta as considerações finais deste trabalho.

\section{Eficiência energética no Brasil}

Conservar energia elétrica significa otimizar a produção, diminuir o consumo de energia e de outros insumos, reduzindo custos, mas sem perder a qualidade dos serviços e a eficiência (Mamede Filho, 1998). De acordo com a IEI (International Energy Initiative), a eficiência energética pode ser definida como a relação entre a energia útil, isto é, aquela realmente convertida na forma desejada para o uso final, e a energia consumida pelo 
(CC BY 4.0) | ISSN 2525-3409 | DOI: http://dx.doi.org/10.33448/rsd-v9i7.3957

equipamento, ou conjunto de equipamentos, realizando essa conversão energética. Aparelhos energeticamente mais eficientes são aqueles que gastam menos energia para cumprir o seu propósito (IEI, 2019).

A partir dos choques do petróleo da década de 1970 e das discussões ambientais da década de 1990, a eficiência energética ganhou projeção mundial e passou a fazer parte da política energética de inúmeros países da Europa, Estados Unidos, Brasil e outros (GESEL, 2011). Os investimentos em métodos capazes de prover energia de forma mais eficiente foram valorizados, permitindo uma redução da demanda e dos gastos com energia, assim como permitiu a viabilidade econômica de projetos de uso eficiente de energia (Chaves \& Tosta, 2016).

Ao longo das décadas, foram implementados vários programas de eficiência energética no Brasil, sendo um dos principais, o Programa de Eficiência Energética (PEE). Estabelecido a partir da Lei no 9.991/2000, seu objetivo é promover o uso eficiente da energia elétrica em todos os setores da economia, por meio de projetos que demonstrem a importância e a viabilidade econômica da melhoria da eficiência energética de equipamentos, processos e usos finais de energia (MME, 2018).

Em 17 de outubro de 2001 foi promulgada a Lei $n^{\circ} 10.295$ de Eficiência Energética, que dispõe sobre a Política Nacional de Conservação e Uso Racional de Energia, estabelecendo níveis máximos de consumo específico de energia, ou mínimos de eficiência energética, de máquinas e aparelhos fabricados ou comercializados no país. Uma das ações dessa política foi iniciada em junho de 2012, com a gradativa retirada das lâmpadas incandescentes ineficientes do mercado, começando pelas de potência maior que 100 Watts. Assim, foram previstos benefícios energéticos da ordem $10 \mathrm{TWh} / \mathrm{ano}$, equivalendo à expansão de $2.433 \mathrm{MW}$ na oferta, proporcionando a economia de aproximadamente R\$ 6 bilhões em custos de geração, transmissão e distribuição (CEPEL, 2014).

Com relação à otimização da energia demandada com a iluminação pública, o governo possui três programas específicos que apoiam projetos com uso de tecnologias e recursos mais eficientes, a saber:

- $\quad$ Programa de Eficiência Energética (PEE) na iluminação pública é executado pelas distribuidoras de eletricidade e coordenado pela ANEEL, com a finalidade apoiar as prefeituras municipais na melhoria da eficiência energética dos sistemas de iluminação pública, com foco no uso de lâmpadas e equipamentos mais eficientes, podendo envolver a troca de reatores, ignitores, luminárias, relés fotoelétricos, fiação, braços, postes e demais elementos de fixação (MME, 2018). 
- $\quad$ Programa Nacional de Iluminação Pública e Sinalização Semafórica Eficientes (PROCEL RELUZ) é executado pela Eletrobrás e parceiros, e coordenado pelo Ministério de Minas e Energia. Foi criado em 2000 e atua no apoio às prefeituras no planejamento e implantação de projetos de sistemas eficientes de iluminação pública e sinalização semafórica, bem como a valorização dos espaços públicos urbanos, melhorando a segurança da população. Em 2017 foi publicada a primeira chamada pública do PROCEL RELUZ, específica para seleção de projetos de eficiência energética em iluminação pública viária com tecnologia LED (MME, 2018).

- Linha de financiamento para Iluminação Pública do BNDES (Banco Nacional de Desenvolvimento) que possui políticas operacionais estabelecendo que o setor de iluminação pública seja uma das prioridades, por trazer impactos positivos nos aspectos sociais, econômicos e ambientais, com ganhos em segurança pública, eficiência energética e redução de custos (MME, 2018).

A participação dos municípios nos programas de eficiência energética da iluminação pública no país, são por meio de chamadas públicas que têm critérios para avaliar e aprovar cada projeto, como a relação custo-benefício da proposta, o potencial em diminuir a demanda e o consumo de energia elétrica, investimento, qualidade, capacidade de mercado, divulgação, entre outros (MME, 2018).

As prefeituras participantes das chamadas públicas nos programas de eficiência energética do país podem enquadrar a aquisição de materiais e serviços necessários à execução de seus projetos na chamada licitação sustentável, que trata-se de uma solução para integrar considerações ambientais e sociais em todos os estágios do processo da compra e contratação dos agentes públicos com o objetivo de reduzir impactos à saúde humana, ao meio ambiente e aos direitos humanos (ICLEI \& GVces, 2019).

\section{Principais recursos de iluminação pública}

Como citado na seção anterior, no Brasil existem programas governamentais focados em projetos de eficiência energética para a iluminação pública por meio do uso de recursos e equipamentos mais eficazes.

Carli, Dotoli e Pellegrino (2019) relatam que, como objetivos principais na tomada de decisão para projetos luminotécnicos da atualidade estão a eficiência energética relacionada ao desempenho, o índice de reprodução de cores dos equipamentos, além da sustentabilidade ambiental. 
Research, Society and Development, v. 9, n. 7, e606973957, 2020

(CC BY 4.0) | ISSN 2525-3409 | DOI: http://dx.doi.org/10.33448/rsd-v9i7.3957

O sistema de iluminação pública utilizado atualmente perdura desde 1960, período no qual as lâmpadas de vapor de sódio e mercúrio passaram a iluminar as cidades brasileiras. Esta iluminação é caracterizada por elevada necessidade de manutenção (Aguera, 2015). Assim, é necessário a revitalização dos projetos luminotécnicos da iluminação pública no Brasil, combinando lâmpadas, reatores e refletores eficientes, bem como o uso tecnologias baseadas em softwares e energias renováveis. Esta seção tem a intenção de apresentar os principais recursos utilizados na iluminação pública no Brasil e suas particularidades.

Com relação às lâmpadas, os diferentes tipos utilizados na iluminação pública ao redor do mundo são as lâmpadas de vapor de sódio de alta pressão (HPSV), as lâmpadas de iodetos metálicos (MH), as lâmpadas de vapor de mercúrio (MV) e as lâmpadas de diodos emissores de luz (LED) (Carli et al., 2016).

O Quadro 1 consolida os principais equipamentos aplicados na iluminação pública relacionando suas características e aplicações mais comuns.

Quadro 1: Principais equipamentos aplicados na iluminação pública.

\begin{tabular}{|c|c|}
\hline EQUIPAMENTO & CARACTERÍSTICAS E APLICAÇÕES \\
\hline $\begin{array}{c}\text { Lâmpada a vapor de } \\
\text { mercúrio em alta } \\
\text { pressão }\end{array}$ & 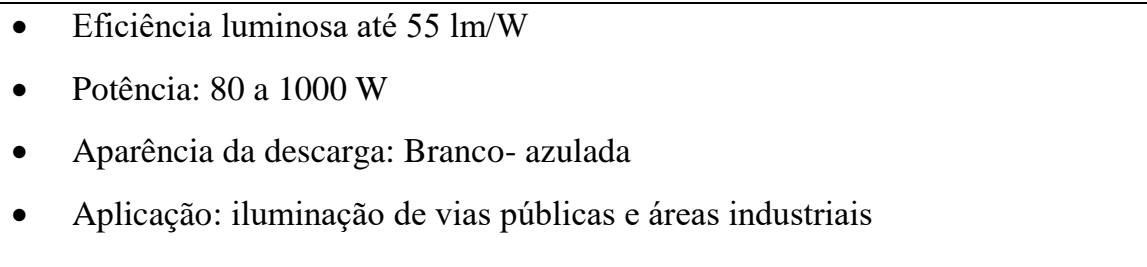 \\
\hline $\begin{array}{l}\text { Lâmpada a vapor de } \\
\text { sódio em alta pressão }\end{array}$ & 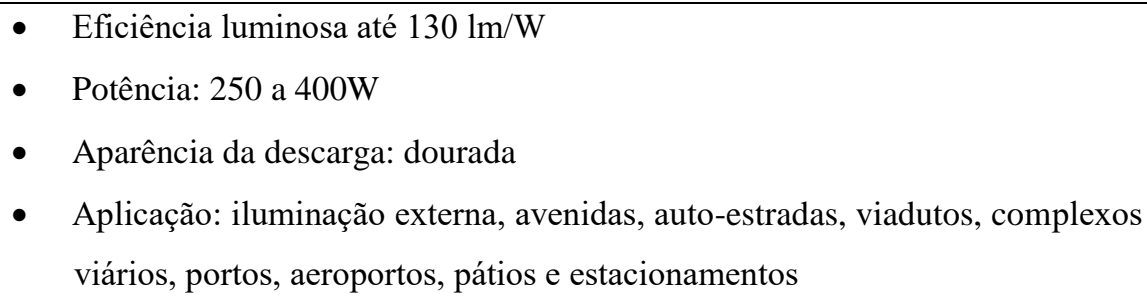 \\
\hline $\begin{array}{c}\text { Lâmpada } \\
\text { multivapores } \\
\text { metálicos de alta } \\
\text { potência }\end{array}$ & $\begin{array}{l}\text { - } \text { Eficiência luminosa até } 100 \mathrm{~lm} / \mathrm{W} \\
\text { - } \\
\text { - } \\
\text { - } \\
\text { - } \\
\text { Aptência: } 250 \text { a } 3500 \mathrm{~W} \\
\text { plicação: iluminação de grandes áreas }\end{array}$ \\
\hline Lâmpada de LED & $\begin{array}{l}\text { - } \text { Eficiência luminosa: } 50 \text { a } 2501 \mathrm{~lm} / \mathrm{W} \\
\text { - } \\
\text { - } \quad \text { Aptência: } 40 \text { a } 180 \mathrm{~W} \\
\text { - } \\
\text { Aplicação: semáforos de trânsito, na iluminação interna de automóveis e em } \\
\text { outros equipamentos de sinalização. Já existem iniciativas de utilização em }\end{array}$ \\
\hline
\end{tabular}




\begin{tabular}{|c|c|}
\hline (diodo emissor de luz) & iluminação pública \\
\hline Reatores & $\begin{array}{l}\text { Provocam um aumento de tensão durante a ignição e uma redução na intensidade da } \\
\text { corrente, durante o funcionamento da lâmpada. Os reatores eletrônicos são os mais } \\
\text { procurados por profissionais voltados ao uso eficiente da energia. }\end{array}$ \\
\hline Dimmer & $\begin{array}{l}\text { Tem como função variar continuamente a intensidade da luz de acordo com a } \\
\text { necessidade. }\end{array}$ \\
\hline Circuitos de comando & $\begin{array}{l}\text { O comando da iluminação é realizado por relés fotoelétricos, que tem por função } \\
\text { identificar o nível de iluminamento natural e acionar ou desativar as lâmpadas, } \\
\text { conforme o período do dia, noturno e diurno. }\end{array}$ \\
\hline Luminárias & $\begin{array}{l}\text { Abriga e fixa a lâmpada, e direciona a luz. Suas partes principais são: receptáculo } \\
\text { para a fonte luminosa, refletores, difusores e carcaça. }\end{array}$ \\
\hline Braço de apoio & $\begin{array}{l}\text { Sustenta as luminárias e serve como eletroduto na proteção dos cabos de alimentação. } \\
\text { Seu ângulo de fixação influencia diretamente na correta distribuição do fluxo } \\
\text { luminoso. }\end{array}$ \\
\hline
\end{tabular}

Fonte: Eletrobras /Procel, 2011; Kruger \& Ramos, 2016; Rodrigues, 2017; Shahzad, 2018.

Os equipamentos listados no Quadro 1, são os mais utilizados nesse tipo de serviço e que podem ser adequados e melhorados para que a iluminação pública se torne mais eficiente. É estimado que a instalação de soluções de iluminação avançada, ao invés de dispositivos de iluminação tradicional, possibilite a economia de até 40\% de energia (Shahzad et al., 2017).

\subsection{Retrofit}

O retrofit é uma das formas mais comuns de promover a redução no consumo de energia com a iluminação pública. E consiste em realizar alterações ou reformas nos sistemas consumidores de energia elétrica. Como ações do retrofit nos sistemas de iluminação pública, é bastante comum a substituição de lâmpadas, conjuntos óticos, acionadores e reatores por outros energeticamente mais eficientes. O processo de aprovação de um retrofit em sistemas de iluminação abrange a análise da tecnologia existente, os benefícios fornecidos pela nova 
(CC BY 4.0) | ISSN 2525-3409 | DOI: http://dx.doi.org/10.33448/rsd-v9i7.3957

solução e a viabilidade econômica fornecida nesta troca (Rodrigues, 2017).

Os meios para que ocorra a eficiência energética na iluminação pública vão além da substituição das lâmpadas convencionais por lâmpadas de LED. Entretanto, o retrofit nos sistemas de iluminação para lâmpadas de LED já é um grande avanço para a conservação dos recursos energéticos.

Conforme relatado na seção anterior, os sistemas de iluminação pública brasileira são antigos e demandam constantes manutenções. Pesquisas da EPEC (European PPP Expertise Centree) de 2013 mostram que os custos com iluminação pública tradicional ao longo de 25 anos é dividido da seguinte forma: $85 \%$ de manutenção/operação e $15 \%$ de custo de capital, aproximadamente (EPEC, 2013). Já os LEDs proporcionam baixo custo de manutenção e substituição, pois possuem um tempo de vida útil médio de 12 anos. Considerando que ficam acesos, em média, de 11 a 12 horas ao dia, a economia de energia elétrica é de $40 \%$ a $50 \%$, proporcionando uma redução significativa do consumo no pico da demanda do setor elétrico (Martinez \& Novicki, 2008).

Dados do Ministério de Minas e Energia (MME), apresentados na Tabela 1, ilustram o comparativo das lâmpadas mais utilizadas na iluminação pública tradicional com as lâmpadas de LED.

Tabela 1: Comparação das lâmpadas aplicadas na iluminação pública.

\begin{tabular}{|c|c|c|c|c|}
\hline $\begin{array}{l}\text { Tipo de } \\
\text { lâmpada }\end{array}$ & $\begin{array}{l}\text { Eficiência luminosa } \\
\qquad(\operatorname{lm} / \mathbf{W})\end{array}$ & $\begin{array}{c}\text { Índice de } \\
\text { reprodução de } \\
\text { cor (IRC) }\end{array}$ & $\begin{array}{l}\text { Vida útil da } \\
\text { lâmpada (h) }\end{array}$ & Preço (R\$) \\
\hline $\begin{array}{l}\text { Vapor de sódio } \\
\text { de alta pressão }\end{array}$ & $80-150$ & 24 & $15.000-24.000$ & 316,00 \\
\hline Vapor metálico & $70-130$ & 96 & $8.000-12.000$ & 320,00 \\
\hline $\begin{array}{l}\text { Vapor de } \\
\text { mercúrio }\end{array}$ & $35-65$ & 40 & $10.000-15.000$ & 285,00 \\
\hline LED & $70-160$ & $70-90+$ & $40.000-90.000$ & $1.500,00$ \\
\hline
\end{tabular}


Através da Tabela 1 é possível observar a durabilidade superior das lâmpadas de LED, o que influencia principalmente nos custos com a manutenção, já que a necessidade de substituir as lâmpadas será consideravelmente menor.

Shahzad et al. (2018) consideraram o impacto que alguns tipos de lâmpadas utilizadas na iluminação pública causam no meio ambiente, e a análise feita por aqueles autores revelou que os impactos ecológicos são de $87 \%$ e $96 \%$ para as luminárias LED e HPS (vapores de sódio de alta pressão), respectivamente. Entretanto, foi relatado pelos autores que as principais vantagens do uso de luminárias LED em comparação com as luminárias convencionais incluem a redução da emissão de carbono, bem como a ausência de mercúrio.

Dentre as propostas de retrofit de equipamentos de iluminação pública, tem-se ainda o aumento da eficiência energética da iluminação pública baseado no controle dos níveis de luminosidade, com a utilização da dimerização (Souza, 2014; Deleuil, 2009; Djuretic \& Kostic, 2018); e a utilização de sistemas autônomos que integram fontes solares aos postes de iluminação pública (Schuch et al., 2011; Santos, 2018; Oliveira, 2017).

$\mathrm{Na}$ Seção 3.2 são detalhadas as aplicações das tecnologias mais recentes em estudos de caso e em projetos de iluminação pelo mundo.

\subsection{Tecnologias aplicadas em projetos de iluminação pública}

Uma das publicações mais recentes utiliza um sistema inteligente de iluminação de vias baseado em redes neurais artificiais implementado em uma área residencial de Hosur, na Índia. Foi observado que a rede neural artificial e o método lógico Fuzzy são eficientes na tomada de decisões para a utilização baseada na demanda, evitando o uso desnecessário das luzes. O sistema de iluminação pública de eficiência energética e inteligente reduziu a utilização indesejada em $34 \%$ e reduziu ainda a taxa de consumo de energia em 13,5\% (Mohandas, Anni \& Gao, 2019). Embora os autores ressaltem que se trata de um estudo inicial, sem apresentar informações de cunho econômico como, por exemplo, custo de investimento, é ainda um bom exemplo de como a aplicação de tecnologia já existente pode aumentar a eficiência energética da iluminação pública.

Os estudos de caso acerca do retrofit dos sistemas para luminárias LED são comuns em várias pesquisas. Os pesquisadores italianos Carli, Dotoli e Pellegrino (2019), abordaram o planejamento ótimo de retrofit nos sistemas de iluminação pública na cidade de Bari, na Itália. Foram usados experimentos numéricos para investigar e quantificar os efeitos do uso de uma abordagem de planejamento multi-período em vez de uma abordagem de período único, 
mostrando maior economia de energia e maior benefício financeiro obtido pelo método proposto para o desenvolvimento do plano ideal de intervenções de adaptação de energia na iluminação pública urbana.

Já os pesquisadores Campisi, Gitto e Morea (2018) avaliaram a viabilidade econômica de melhorias na eficiência energética em sistemas de iluminação pública em Roma. A implementação do sistema de LED em Roma poderia economizar 44.451,10 tCO2 equivalente por ano. Foi avaliada a economia de custos do projeto, considerando a incerteza do preço da eletricidade e do investimento em múltiplos estágios. Os resultados gerais mostram que o projeto é economicamente viável. Em particular, um investimento de cerca de $€ 209$ milhões gera um valor presente líquido (VPL) de cerca de $€ 23,6$ milhões, um retorno sobre o investimento (ROI) de cerca de 1,1 e uma taxa interna de retorno (TIR) de cerca de 9\%. Já o tempo de retorno simples (SPT) é de 8 anos.

Pesquisadores da Sérvia, Djuretic e Kostic (2018), realizaram a substituição de lâmpadas de vapor de sódio de alta pressão (HPS) por luminárias LED em uma rua, na cidade de Belgrado, desenvolvendo um esquema que superasse a deficiência de estudos anteriores, que estabeleciam economias reais de energia com a substituição. A abordagem foi baseada nos níveis de luminância fotópica e mesópica iguais nas instalações de iluminação pública com LED e HPS, estabelecendo-se regimes de iluminação padrão e reduzida nas luminárias LED, permitindo a determinação do seu potencial de economia de energia em vários cenários de dimerização. Concluiu-se que, quando se utiliza LED em vez de luminárias HPS, a economia de energia pode situar-se entre $31 \%$ e $60 \%$, quando se aplicam cenários de escurecimento em várias fases.

As abordagens das pesquisas relatadas avaliaram variáveis distintas do retrofit das luminárias tradicionais por LED e em todas elas os resultados foram positivos, o que corrobora com os dados já referenciados. O retrofit das luminárias tradicionais por LED é totalmente possível para os municípios brasileiros, principalmente pelo fato de existirem programas governamentais de financiamento aos projetos de eficiência energética da iluminação pública.

No Brasil, também são realizados estudos de caso com aplicação de tecnologias na iluminação pública urbana. Dois trabalhos que se destacam, utilizaram um microcontrolador por placa de Arduino.

Clemente et al. (2018) desenvolveram um protótipo de monitoramento para atendimento à Companhia Energética de Minas Gerais (CEMIG) para constatar a economia de um ponto de iluminação pública convencional composto por luminárias de vapor de 
mercúrio ao ser substituída por luminárias de LED. Foi possível monitorar a potência e corrente em tempo real dos protótipos. A luminária de vapor de mercúrio apresentou valores de pico de corrente e de potência em seu momento inicial de trabalho até que seu fluxo luminoso fosse estabilizado, enquanto a luminária de LED apresentou valores constantes desde seu início de trabalho, incorrendo em menor consumo de energia. Os dados foram analisados por gráficos gerados pelas variáveis monitoradas pelo microcontrolador. Por meio desse sistema, também é possível avaliar a necessidade de manutenção do ponto escuro através da análise da potência monitorada no referido ponto, sendo emitida uma mensagem de alerta informando a necessidade de troca da luminária. Esse projeto demonstrou o quanto a luminária de LED proporciona autonomia sobre o controle do sistema de iluminação pública.

Já Fonseca et al. (2016) apresentaram um estudo de caso realizado na cidade de Caruaru/PE, utilizando o microcontrolador por placa de Arduino para melhorar os tempos de atuação dos relés fotoelétricos, controlando a quantidade de fluxo luminoso através da automatização dos relés das lâmpadas de vapor de mercúrio com potência de $250 \mathrm{~W}$. Observou-se o potencial e a possível economia de aproximadamente $\mathrm{R} \$ 45.000,00$ por mês com esta proposta de otimização do sistema público de iluminação.

O retrofit também é avaliado em vários estudos de caso pelas cidades brasileiras. Em 2016, no Bairro de Arroio Corrente, na cidade de Jaguaruna/SC, foi realizado um estudo de retrofit de lâmpadas convencionais por lâmpadas de LED. Luiz (2016) propôs a substituição de 422 luminárias do bairro, sendo a maior parte de vapor de sódio, por lâmpadas do tipo LED de $70 \mathrm{~W}$ e $90 \mathrm{~W}$. Foi elaborado um projeto de eficiência luminotécnica atendendo à norma de iluminação pública NBR5101 e atendendo aos parâmetros da ANEEL (Agência Nacional de Energia Elétrica). Com base no estudo, Luiz (2016) concluiu que o projeto é viável para aplicação, gerando economia de energia em torno de $23 \%$ ao ano.

Outro grupo, composto pelos pesquisadores Kruger e Ramos (2016), averiguou quatro modelos de lâmpadas utilizadas na iluminação pública, conforme demonstrado na Tabela 2, e constatou que a lâmpada de LED é a mais indicada, no que se refere ao consumo de energia, custo-benefício, eficiência luminosa, fluxo luminoso e vida útil. 
Tabela 2: Comparativo do gasto mensal das lâmpadas.

\begin{tabular}{lccccc}
\hline \multicolumn{1}{c}{ Lâmpada } & Potência $(\mathbf{W})$ & Horas & Dias & $\mathbf{k W . h} / \mathbf{m}$ & Gasto Mensal (R\$) \\
\hline LED & 150 & 10 & 30 & 23,40 & $23.400,00$ \\
Multivapores metálicos & 250 & 10 & 30 & 39,00 & $39.000,00$ \\
Vapor de sódio & 250 & 10 & 30 & 39,00 & $39.000,00$ \\
Vapor de mercúrio & 400 & 10 & 30 & 62,40 & $62.400,00$ \\
\hline
\end{tabular}

Fonte: Adaptada de Kruger \& Ramos (2016).

A avaliação do gasto mensal com iluminação resultou que o LED é mais vantajoso, conforme observado na Tabela 2, onde é apresentado o gasto mensal médio de cada tipo de lâmpada. Os valores são aproximados, pois foi considerado o tempo de 10 horas diárias de utilização dos equipamentos, o que pode variar dependendo da estação do ano. Foi considerada a taxa de $\mathrm{R} \$ 0,52 / \mathrm{kWh}$, baseada na tabela de março/ 2016 da RGE Sul (distribuidora de energia elétrica para a região Centro-Oeste do Rio Grande do Sul, antiga AES Sul).

Existem ainda projetos avançados que integram fontes eólicas ou fotovoltaicas aos postes de luz, dispensando redes e outros equipamentos. Devido à baixa tensão dos LEDs, é possível a conexão às baterias de acumuladores, dispensando o auxílio da rede comum de tensão (Gesel, 2011).

Schuch et al. (2011), Santos (2018) e Oliveira (2017) propuseram sistemas autônomos de iluminação pública baseado em energia solar. Os sistemas apresentam-se como uma alternativa viável para localidades isoladas e de difícil acesso, bem como para estradas e trevos; e podem ser adotados como alternativa para iluminação pública em centros urbanos ou atuar como iluminação de emergência. Assim, além de viabilizar iluminação aos locais que ainda não são interligados às linhas de transmissão, podem gerar economia no consumo de energia elétrica na iluminação pública, utilizando o potencial solar das localidades com inexpressivos impactos ao meio ambiente.

A implantação desse tipo de sistema requer valor elevado de investimentos e necessidade de substituição dos postes e luminárias, o que gera uma quantidade considerável de resíduos. No entanto, em algumas localidades, como Campinas/ SP e Criciúma/ SC, já existem postes solares em funcionamento atendendo à iluminação de espaços públicos.

A dimerização é outro recurso utilizado para melhoria da eficiência energética na 
(CC BY 4.0) | ISSN 2525-3409 | DOI: http://dx.doi.org/10.33448/rsd-v9i7.3957

iluminação pública com o uso de reatores eletrônicos que permitem a regulação do fluxo luminoso e oferecem uma economia considerável de energia, desde que a iluminação esteja atrelada a um sistema de controle automático, com a detecção dos níveis de iluminação e ajuste do fluxo luminoso da lâmpada, de forma a manter seu nível constante. A iluminação pode ser programada para diminuição do fluxo luminoso quando determinadas áreas não estão sendo ocupadas (Souza, 2014; Deleuil, 2009).

Deleuil (2009) realizou um experimento utilizando dimerização nos pontos de luz de algumas ruas da cidade de Lyon, na França, provando que a redução dos níveis de iluminação pública de forma controlada é perfeitamente plausível e não afeta o cotidiano ou o conforto visual da população em geral, trazendo uma maior economia nos gastos com a energia elétrica municipal. A iluminação pública de Lyon consome anualmente cerca de 40 milhões de kWh. Estima-se que a economia provocada por uma redução de 30\% do nível de toda a iluminação pública da cidade representaria 6,5 milhões de $\mathrm{kWh}$, ou seja, cerca de 16,2\% de economia.

A fim de resumir os recursos e tecnologias apresentados para se obter eficiência energética na iluminação pública urbana, foi construído o Quadro 2, englobando as vantagens e desvantagens de cada tipo discutido neste trabalho. 
Research, Society and Development, v. 9, n. 7, e606973957, 2020

(CC BY 4.0) | ISSN 2525-3409 | DOI: http://dx.doi.org/10.33448/rsd-v9i7.3957

Quadro 2: Resumo das tecnologias apresentadas para iluminação pública.

\begin{tabular}{|c|c|c|}
\hline Recurso/ Tecnologia & Vantagens & Desvantagens \\
\hline Retrofit LED & $\begin{array}{l}\text { - Maior durabilidade das luminárias; } \\
\text { - Menor consumo de energia; } \\
\text { - Possibilidade de integrar num sistema } \\
\text { automatizado } \\
\text { - Ausência de mercúrio nas lâmpadas. }\end{array}$ & - Custo inicial do sistema. \\
\hline $\begin{array}{c}\text { Dimerização nos } \\
\text { sistemas automatizados }\end{array}$ & $\begin{array}{l}\text { - Menor consumo de energia em } \\
\text { horários de menor circulação de } \\
\text { pessoas. }\end{array}$ & $\begin{array}{l}\text { - Requer sistema com controle } \\
\text { automático (LED). }\end{array}$ \\
\hline Poste com Fonte Solar & $\begin{array}{l}\text { - Uso em locais de difícil acesso, sem } \\
\text { interligação à rede elétrica; } \\
\text { - Energia gerada por fonte renovável e } \\
\text { limpa. }\end{array}$ & - Custo inicial do sistema. \\
\hline $\begin{array}{c}\text { Microcontrolador por } \\
\text { placa de Arduino }\end{array}$ & $\begin{array}{l}\text { - Ajuste nos tempos de atuação dos } \\
\text { relés fotoelétricos, corrigindo falhas; } \\
\text { - Monitoramento de corrente e potência } \\
\text { das luminárias, oferecendo informação } \\
\text { das luminárias danificadas. }\end{array}$ & $\begin{array}{l}\text { - Trata-se apenas de um recurso } \\
\text { para melhorar o sistema } \\
\text { existente. }\end{array}$ \\
\hline $\begin{array}{c}\text { Uso de redes neurais } \\
\text { artificiais }\end{array}$ & $\begin{array}{l}\text { - Total controle do sistema de } \\
\text { iluminação pública. }\end{array}$ & $\begin{array}{l}\text { - Estudo muito recente, sem } \\
\text { informações de custos para os } \\
\text { projetos; } \\
\text { - Necessidade de sistema } \\
\text { baseado em luminárias LED. }\end{array}$ \\
\hline
\end{tabular}

Fonte: Elaborada pelos autores (2020).

\section{Considerações Finais}

Através do desenvolvimento deste trabalho, foi possível averiguar os principais recursos disponíveis para aumentar a eficiência energética da iluminação pública por meio de 
pesquisa bibliográfica e documental.

De acordo com as pesquisas e dados apresentados, foi possível constatar que os sistemas que mais se enquadram para as características das cidades brasileiras são o que utilizam a tecnologia LED. O sistema eficiente e mais simples de ser adotado é o retrofit das luminárias de LED, que, apesar dos valores altos de investimentos, gera um retorno positivo na economia de energia, na redução dos custos com manutenção e nos menores impactos ambientais devido à ausência do mercúrio nesse tipo de lâmpada, além da menor geração de resíduos já que a durabilidade das lâmpadas de LED é consideravelmente superior à tecnologias mais antigas.

Outro sistema atrativo, sobretudo para as regiões sem interligação com a rede elétrica, é o baseado em postes com fontes solares. No entanto, os custos de implantação são demasiadamente altos, mas a maior das vantagens, além da economia nos custos com eletricidade a longo prazo, é o fato de se tratar de uma fonte limpa e renovável, ainda mais se tratando de um país tropical que possui grande incidência de radiação solar na maior parte do ano, como o Brasil.

O microcontrolador por placas de Arduino atua como auxiliar no monitoramento do sistema de iluminação pública e pode ser implantado nos sistemas baseados em luminárias convencionais e LED, e deve ser avaliado por cada gestor a viabilidade de sua implantação.

As demais tecnologias auxiliares, dimerização e redes neurais artificiais, requerem a implantação do sistema LED. Desta forma, a utilização dessas tecnologias demanda custos também com a implantação de um sistema preliminar, o que não se torna atrativo para a realidade brasileira.

Todos os recursos e tecnologias relatados são formas de contribuir para a redução do consumo de energia elétrica pela iluminação pública, consequente diminuição dos gastos com energia elétrica pelas prefeituras, da demanda por eletricidade e do consumo dos recursos naturais, provocando assim menores impactos ambientais causados pela geração de energia elétrica.

\section{Agradecimentos}

O presente trabalho foi realizado com apoio da Coordenação de Aperfeiçoamento de Pessoal de Nível Superior - Brasil (CAPES) - Código de Financiamento 001. 


\section{Referências}

Aguera, R. S. (2015). Cenário brasileiro da iluminação pública. São Carlos: USP. Retrieved Nov 23, 2019, from www.tcc.sc.usp.br > tce > disponiveis > publico > Aguera_Roger_Saraivatcc

Campisi, D., Gitto, S. \& Morea, D. (2018). Economic feasibility of energy efficiency improvements in street lighting systems in Rome. Journal of Cleaner Production, v. 175, 190-198. doi: 10.1016/j.jclepro.2017.12.063

Carli, R., Dotoli, M., Andria G. \& Lanzolla, A. M. L. (2016). Bi-level programming for the strategic energy management of a smart city. In: IEEE Workshops on Environmental, Energy, and Structural Monitoring Systems, 1-6. Retrieved Nov 10, 2019, from https://ieeexplore.ieee.org/document/7504820

Carli, R., Dotoli, M. \& Pellegrino, R. (2019). A Multi-Period Approach for the Optimal Energy Retrofit Planning of Street Lighting Systems. Applied Sciences, v. 9(5), 1025. doi: 10.3390/app9051025

CEPEL. Guia para eficientização energética nas edificações públicas: Centro de Pesquisas de Energia Elétrica (2014). Retrieved Nov 23, 2019, from http://www.mme.gov.br/documents/10584/1985241/GUIA+EFIC+ENERG+EDIF+PUBL_1+ 0_12-02-2015_Compacta.pdf

Chaves, G. L. D. \& Tosta, M. C. R. (2016). Gestão de sistemas de energia. Curitiba: CRV.

Clemente, A. C., Paiva, C., Junior, W. C. M. \& Caixeta, I. N. (2018). Gerenciamento de iluminação pública. Revista Científica Multidisciplinar Núcleo do Conhecimento, v. 05, 107147. Retrieved Nov 22, 2019, from https://www.nucleodoconhecimento.com.br/engenhariaeletrica/iluminacao-publica

Dapper, P. V., Tomé, B. P. \& Zanatta, J. M. (2019). Eficiência energética: estudo de caso em agroindústria do Rio Grande do Sul. Research, Society and Development. v. 09 (2), 1-35. doi: $10.33448 /$ rsd-v9i2.2042 
Deleuil, J. M. (2009). De L'acceptabilité Socialedes Diminuitions D’éclairement: Une Expérimentation Lyonnaise. In: Eclairer La Ville Autrement: Innovations et Expérimentations em Ecláirage Public. Presses Polytechniques et Universitaires Romandes. Retrieved Out 06, 2019, from https://halshs.archives-ouvertes.fr > halshs-01144019

Djuretic, A. \& Kostic, M. (2018). Actual energy savings when replacing high-pressure sodium with LED luminaires in street lighting. Energy, v.157, 367-378. doi:

10.1016/j.energy.2018.05.179

Elektro, UNIFEI, EXCEN \& FUPAI (2012). Eficiência Energética: fundamentos e aplicações. Campinas. Retrieved Nov 25, 2019, from

https://www.voltimum.com.br/sites/www.voltimum.com.br/files/pdflibrary/01_livro_eficienci a_energetica.pdf

Eletrobras/ Procel (2011). Manual de iluminação no Brasil. Retrieved Out 22, 2019, from http://www.mme.gov.br/documents/10584/1985241/MANUAL+DE+ILUMINACAO++PROCEL_EPP+-AGOSTO+2011.pdf/d42d2f36-0b90-4fe0-805f54b862c9692c;jsessionid=A7AE9AD7FFE410D97E371853D50763B0.srv154

EPEC (2013). Energy Efficient Street Lighting: European PPP Expertise Centree. Retrieved Out 25, 2019, from https://ppp.worldbank.org/public-private-partnership/library/energyefficient-street-lighting-epec

Fonseca, D.C.F, Alves, U. E. F., Matos, F. A. G., Silva, M. K. A. \& Silva, I. G. A. M. (2016). Eficiência energética da iluminação pública automatizada: estudo de caso na cidade de Caruaru. In: XXXVI Encontro Nacional de Engenharia de Produção. Retrieved Out 21, 2019, from http://www.abepro.org.br/biblioteca/TN_STO_234_364_29766.pdf

GESEL (2011). Eficiência energética na iluminação pública e o plano nacional de eficiência energética: Grupo de Estudos do setor elétrico. Rio de Janeiro: UFRJ. Retrieved Nov 19, 2019, from http://www.gesel.ie.ufrj.br/app/webroot/files/publications/48_TDSE42.pdf 
ICLEI - European Secretariat GmbH \& GVces - Centro de Estudos em Sustentabilidade da Escola de Administração de Empresas de São Paulo da Fundação Getúlio Vargas. Guia de compras públicas sustentáveis: Uso do poder de compra do governo para a promoção do desenvolvimento sustentável. Retrieved Out 22, 2019, from http://www.mma.gov.br/estruturas/a3p/_arquivos/guia_compras_sustentaveis.pdf

IEA - International Energy Agency (2019). Electricity Information 2019. Retrieved Abr 24, 2020, from https://www.iea.org/reports/electricity-information-2019

IEI - International Energy Initiative (2019). Projeto Geração distribuída, eficiência energética e o consumidor final: propostas para a realidade brasileira. Retrieved Nov 23, 2019, from https://iei-brasil.org/geracao-distribuida-eficiencia-energetica-e-o-consumidorfinal-propostas-para-a-realidade-brasileira/

Kruger, C. \& Ramos, L.F. (2016). Iluminação pública e eficientização energética. Revista Espaço Acadêmico, n. 185, 37-49, 2016. Retrieved Nov 10, 2019, from http://www.periodicos.uem.br/ojs/index.php/EspacoAcademico/article/view/31530

Luiz, C. C (2016). Estudo de eficiência energética em luminárias destinadas à iluminação pública na cidade de Jaguaruna-SC. Joinville: UDESC. Retrieved Nov 20, 2019, from http://sistemabu.udesc.br/pergamumweb/vinculos/00002a/00002a9b.pdf

Mamede Filho, J. (1998). Economia de Energia Elétrica na Indústria e Comércio. Mundo Elétrico, v. 344, 51-55.

Manzano-Agugliaro, F., Alcayde, A., Montoya, F. G., Zapata- Sierra, A. \& Gil, C. (2013). Scientific production of renewable energies worldwide: An overview. Renewable and Sustainable Energy Reviews, v. 18, 134-143. doi: 10.1016/j.rser.2012.10.020

Martinez, R. \& Novicki, J. M. (2008). Leds para iluminação pública. Curitiba: UFPR. Retrieved Nov 21, 2019, from http://www.cricte2004.eletrica.ufpr.br/ufpr2/tccs/41.pdf

MME - Ministério de Minas e Energia (2018). Iluminação pública municipal: Programas e políticas públicas. Retrieved Nov 21, 2019, from 
http://www.mme.gov.br/documents/10584/0/Livreto+Iluminação+Pública_2018_02_19.pdf/b 47c5c44-eebd-4556-ab10-53a1bbba1a42

Mohandas P., Anni, J. S. \& Gao, X. Z. (2019). Artificial Neural Network based Smart and Energy Efficient Street Lighting System: A Case Study for Residential area in Hosur. Sustainable Cities and Society, v. 48, 101499. doi: 10.1016/j.scs.2019.101499

Oliveira, B. V. B. (2017). Estudo de Viabilidade para Instalação de um Poste de Iluminação Considerando um Sistema Fotovoltaico Autônomo. Londrina: UEL. Retrieved Out 05, 2019 from http://www.uel.br/ctu/deel/TCC/TCC2017_BrunoVillasBoasOliveira.pdf

Rodrigues, F. (2017). Eficiência energética aplicada em sistemas de iluminação pública. Novo Hamburgo: UFSM. Retrieved Out 25, 2019 from

https://repositorio.ufsm.br/bitstream/handle/1/12208/TCCE_EEAPP_EaD_2017_RODRIGUE S_FERNANDO.pdf?s

Santos, K. B. (2018). A viabilidade e eficiência da energia solar fotovoltaica para o equipamento público de iluminação. In: VII Congresso Brasileiro de Energia Solar. Retrieved Out 15, 2019 from https://anaiscbens.emnuvens.com.br/cbens/article/view/319

Schuch, L., Costa, M. A. D., Michels, C. R. L., Costa, G. H. \& Santos, A.S. (2011). Sistema autônomo de iluminação pública de alta eficiência baseado em energia solar e LEDs. Revista Eletrônica de Potência, v. 16, 17-27. Retrieved Nov 05, 2019 from https://sobraep.org.br/artigo/sistema-autonomo-de-iluminacao-publica-de-alta-eficienciabaseado-em-energia-solar-e-leds/

Shahzad, K., Cucek, L., Sagir, M., Ali N., Rashid, M. I., Nazir, R., Nizami, A. S., Al-Turaif, H. A. \& Ismail, I. M. I. (2018). An ecological feasibility study for developing sustainable street lighting system. Journal of Cleaner Production, v. 175, 683-695. doi: 10.1016/j.jclepro.2017.12.057

Souza, M. B. (2014). Estratégias para o aumento da eficiência energética em iluminação pública baseadas no controle dos níveis de luminosidade. Ouro Preto: UFOP. Retrieved Out 18, 2019 from 
https://www.em.ufop.br/images/MonografiasControleAutomacao/2014/MarcelBeckmandeSo uza.pdf

\section{Porcentagem de contribuição de cada autor no manuscrito}

Drielly Mazzarim Bernades - 80\%

Wanderley Cardoso Celeste $-10 \%$

Gisele de Lorena Diniz Chaves - 10\% 